(Aus der I. Universitäts-Frauenklinik in Wien. Vorstand: Hofrath Schauta.)

\title{
Ueber einen Fall seltener Missbildung (Hemignathie).
}

Von

\author{
Dr. Oskar Bürger, \\ Assistenten der Klinik.
}

(Mit 2 Abbildungen im Text.)

Je mehr man bei der Ertorschung der Ursachen von angeborenen Entwickelungsanomalien auf mechanische Verhältnisse einzugehen sich bemüht, desto häufiger wird es möglich sein, von aussen einwirkende Momente für die Erklärung dieser abnormen Bildungen heranzuziehen und sich vom hypothetischen Boden der sogenannten inneren Ursachen entfernen zu können. Es bricht sich diese Anschauungsweise vielleicht deshalb so schwer Bahn, weil man nicht gewohnt ist, dieselben mechanischen Einflüsse, denen das extrauterin lebende Individuum ausgesetzt ist, auch auf den Fötus in utero zu übertragen, obwohl auch dieser den verschiedenartigsten von seiner Umgebung herrührenden Einwirkungen, wenn diese vielleicht auch weniger intensiv sind, unterliegt.

Wenn es nun auch für die Mehrzahl der Fälle von angeborener Missbildung gelingen dürfte, eine derartige äussere oder mechanische Ursache supponiren za können, so ist dies doch nicht immer möglich und man wird auf die Vorstellung von primären Bildungsdefecten oder Mangel an Bildungsmaterial zurückgreifen müssen. Deshalb erscheint es wünschenswerth, jeden einzelnen Fall auf Grund genauer Beobachtung und Verarbeitung zur Lösung der Frage heranziehen zu können. Aber auch aus der Zusammenstellung eines grossen klinischen Materiales lassen sich einige Anhaitspunkte, welche nicht unwesentlich erscheinen, gewinnen. 
Eine Durchsicht der Protokolle unserer Klinik aus den Jahren 1892-1901 ergab, dass unter 34329 Geburten 80 Fälle vorkamen, bei denen angeborener Weise Missbildungen oder Entwickelungsstörungen constatirt werden konnten, was einem Verhältniss ron $0,24 \mathrm{pCt}$. entspricht, sodass auf 417 normal gebildete Kinder je 1 Missbildung käme. Diese Zablen sind relativ niedrig, da z. B. von Winckel unter 10056 Kindern der Dresdener Frauenklinik 156 Nissbildungen $=1,3$ pCt: und unter 8149 Kindern der Münchener Klinik sogar $232=2,8$ pCt. findet. Diese Differenz lässt sich theilweise daraus erklären, dass in unserem Materiale die Abnormitäten in der Extremitätenhaltung (Klumpfuss, Pes calcaneus etc.) nicht mitgerechnet sind und dass vielleicht auch geringgradige Abweichungen vom Normalen in den Protokollen nicht zur Notirung kamen. Auch wurden alle Fälle ausgeschaltet, welche Bildungsanomalien untergeordneter Bedentung wie Nävi, Hautanhänge etc. zeigten oder solche, in denen es sich um Neubildungen (Struma, Lymphangioma, Polypen u. a. m.) handelte.

In Bezug auf das Geschlecht der Kinder scheint keine besondere Prävalenz zu walten, denn es finden sich darunter 45 männliche und 31 weibliche Früchte, bei vieren ist das Geschlecht nicht angegeben. Hingegen ist es auffallend, dass trotz der oft hochgradigen Entwickelungsstörungen, welehe auch eine intrauterine normale Entwickelung bis ans Ende oder überhaupt ein Weiterleben schwer erklärlich machen, doch nur eine relativ geringe Anzahl der Früchte (19) die Zeichen der Reife vermissen lassen und viele sogar eine über die Norm weit binausgehende Entwickelung zeigen.

Ein weiterer Umstand, der in Betracht kommt, ist die Erscheinung, dass fast in der Hälfte der Fälle (32) die Anomalien eine Multiplicität zeigen, sodass an einem Individuum Bildungsstötungen versehiedenster Art zu beobachten sind, die untereinander in gar keinem ursächlichem Zusammenhang zu stehen seheinen.

Was nun die einzelnen Arten von Missbildung anlangt, so sind allerdings jene am häufigsten vertreten, für deren Erklärung man mechanische Ursachen wie Oligohydramnie, Amputationen durch Nabelschnurschlingen, frühzeitiges Verwachsen der Amnionkopf kappe, Simonart'sche Bänder und andere Anomalien der Eihüllen heranziehen kann.

So finden sich am häufigsten stärkere Grade von Hydrocephalus (22) und Anencephalus (8), Bildungsanomalien im Bereiche des 
Oberkiefers und Gaumens (26), Syndactylie, Spina bifida und Entwickelungsstörungen im Bereiche des Urogenitalsystemes (Atresia ani, Hypospadie, Kloakenbildung).

Seltener sind die Verkrümmungen und Abknickungen der Extremitäten durch intrauterine Fracturen, wenn solche nicht überhaupt als Folgen intrauteriner Knochenprocesse aufzufassen sind, wie in einem unserer Fälle aus der letzten Zeit, bei dem ich neben verschiedenartigen Missbildungen Veränderungen der histologischen Structur der Knochen nachweisen konnte ${ }^{1}$ ).

Ferner kommen in Betracht Defecte einzelner Extremitäten oder deren Knochen, Narben, Anhänge oder Defecte ganzer Organe.

Fast alle diese Missbildungen lassen eine Erklärung durch mechanische, von aussen wirkende Ursachen zu. Wie aber soll man einen Fall auflösen, bei dem neben grossen Defecten im Bereiche des Schädels und des Gesichtes, Syndactylie, der Bildung cines apfelgrossen Tumors am Hinterhaupte ein vollständiger Defect der einen Niere, des Ureters und der Vagina besteht? (Prot. No. 939 ex 1892).

Ein weiteres Beispicl wäre die Combination von Spina bifida mit Störungen in der Entwickelung der Hirnnerven und Gehirnwindungen, die sich bei der Autopsie ergaben (Prot. No. 3476 ex 1900).

Solche und ähnliche Fälle lassen nur schwer eine Erklärung durch mehanisehe Momente zu und man wird bei ihnen doch mit der Möglichkeit primärer Anlagedefecte rechnen müssen.

Besonderes Interesse dürfte ein Fall haben, welchen ich in der letzten Zeit an der Klinik meines hochverehrten Chefs, Herrn Hofrath Schauta, zu beobachten Gelegenheit hatte und der nicht nur wegen der Multiplicität der Entwickelungsstörungen, sondern auch in Folge der grossen Seltenheit eines der Defecte, wie er in gleichem Maasse bis jetzt überhaupt noch nicht bekannt geworden sein dürfte, ausführlicher behandelt werden soll.

Die Geburtsgeschichte ergiebt kurz Folgendes: Die 34 Jahre alte, verheirathete Frau kam am 11.4. 1902 als Drittgebärende an unsere Klinik. In ihrer Familie sind weder väterlicher- noch mütterlicherseits Missbildungen vorgekommen, ebenso wenig in der Familie ihres Mannes, der jedoch Potator sein soll. Während der

1) Demonstration in der Wiener geburtshülflichen Gesellschaft. Sitzung rom 16. VI. 1902. 
diesmaligen Gravidität ist die Frau angeblich mehrere Male auf die linke Seite gefallen. Im Uebrigen hat sie nie ernstlichere Erkrankungen durchgemacht,

Die Menstruation trat zum ersten Male im 18. Lebensjahre ein, war unregelmässig, manchmal durch längere Zeit cessirend, von viertägiger Dauer, oft mit Schmerzen verbunden. Von den früheren Geburten wurde die erste vor zwei Jahren mittelst Forceps künstlich beendigt, bei der zweiten, vor einem Jahre erfolgten Entbindung handelte es sich um Gemini, die zweite Frucht wurde in Steisslage geboren. Beide Puerperien verliefen afebril. Alle drei Kinder sollen vollständig wohlgebildet gewesen sein. Die Zwillinge starben in frühem Alter, der eine mit vier Wochen an "Flecken", der andere mit acht Wochen an "Darmkatarrh". Letzte Menses am 14. 7. 1901, erste Kindesbewegungen am 15. 11. 1901.

Die bei der Aufnahme der Frau vorgenommene Untersuchung ergab eine Beckenendlage II. Position. Normale Beckenverhältnisse. Cervicalcanal $2 \mathrm{~cm}$ lang, für einen Finger durchgängig; über dem Becken hoch und beweglich der Steiss der Frucht. Es besteht ein leichter Descensus beider Vaginalwände. Nach circa 6stïndiger Wehenthätigkeit erwies sich Cervicalcanal und Muttermund als verstrichen, der Steiss ins Becken eingetreten. Bei der künstlichen Blasensprengung entleerte sich eine der Norm gegenüber vermehrte Menge von Fruchtwasser. Eine Stunde später erscheint der Steiss in der Vulva; es wird in typischer Weise VIanualbülfe geleistet.

Die 5 Minuten post partum spontan abgegangene Placenta, sowie die Eihäute erwiesen sich als vollständig; es sind nirgends abnorme Verdickungen oder Stränge nachweisbar; beide Nabelarterien sind vorhanden.

Die Frucht ist männlichen Geschlechts, lebend $2340 \mathrm{~g}$ schwer, $47 \mathrm{~cm}$ lang. Der Schädel zeigt einen mesocephalen Typus. Die Behaarung der Kopfhaut entspricht der eines reifen Kindes. Abweichend von der Norm ist der grosse Kopfumfang mit $33 \mathrm{~cm}$ und der grosse schräge Durchmesser mit $11,5 \mathrm{~cm}$. Die übrigen Kopfmaasse sind annähernd normal. Die grosse Fontanelle ist $9 \mathrm{~mm}$ breit, die kleine Fontanelle geschlossen, der intracerebrale Druck kaum erhöht. Die Coronarnähte sind stark prominent and verbreitert. Die Bulbi erscheinen protundirt, die Pupillen weit, leichter Strabismus convergens. Der an der Klinik Hofrath Schnabel von Herrn Assistenten Dr. Fröhlich erhobene Augenbefund ergiebt: "Die Bulbi normal gebildet, auch der Fundus vollständig normal. Es besteht Hypernetropie von 1 D. Das Skelett der Orbita ist in seiner oberen Hälfte - das Orbital- 
dach also - missgeformt. Dadurch steht der Hornhautscheitel über den oberen Knochenrand vor, was auf eine abnorme Seichtigkeit der Orbita deutet. Der knöcherne Rand unten ist normal." Die Ohren zeigen eine Verbreiterung und Abflachung des Helix beiderseits. Der äussere Gehörgang ist vorhanden. Die von Herrn Assistenten Dr. Alexander ausgeführte specialistische Untersuchung ergab normale VerhäItnisse. Die untere Gesichtshälfte nun ist deutlich asymmetrisch. Das Kinn nicht vorspringend, sondern abgeflacht, was den Eindruck des sogenannten "Vogelgesichtes" hervorruft.

Bei näherer Besichtigung erkennt man, dass der linke Unterkiefer in normaler Grösse ausgebildet, jedoch in toto nach rechts gedreht und verschoben ist. Der Knochen endigt etwas rechts von der Medianlinie stumpf. Die rechte Unterkieferhälfte fehlt scheinbar vollständig, nur vom Corpus scheint ein kleines Stück in Verbindung mit dem linksseitigen Knochen ausgebildet zu sein, da man innen an dem horizontalen Knochensegmente, wenige Millimeter vor seiner stumpfen Endigung die Andeutung einer sagittalen Knochenleiste (Spina mentalis interna) fühlen kann. Der Angulus mandibulae der rechten Seite fehlt vollständig, ebenso der ganze Ramus ascendens, Processus coronoides und condyloideus. Unter dem rechten Jochbeine ist weder von aussen noch von der Mundhöhle aus eine Gelenkgrube nachweisbar. Die Haut über dem Krochenende des Unterkiefers ist durch subcutane Muskelfasern fixirt, nirgends jedoch eine Narbe in der Haut oder eine tiefere Furche za sehen.

Die Mundöffnung ist auffallend klein, asymmetrisch; die Zunge verkürzt, an ihrer ganzen rechten Seite angewachsen und nach dieser Seite verzogen. Der harte Gaumen ist normal gewölbt, zeigt keinerlei Defect und es ist auch nirgends eine Spaltbildung bemerkbar. Beide Oberkiefer scheinen normal entwickelt.

Am Halse bestehen keine Abnormitäten. Die Mm. sternocleido-mastoidei sind beiderseits gleich gut entwickelt.

Die oberen Extremitäten sind bis zum Ellbogengelenke beide gut ausgebildet. Der Humerus ist beiderseits $8 \mathrm{~cm}$ lang; Schultergürtel und Oberarmmusculatur normal entwickelt. Der rechte Vorderarm zeigt eine Länge von $61 / 2 \mathrm{~cm}$ und im ganzen Verlaufe beide Knochen entwickelt. Dieselben werden im Interossealgelenke activ verschoben, so dass Pro- und Supination anstandslos von 
statten gehen. Auch die beiden Gelenksenden sind gut ausgebildet und tragen die Proçessus styloidei.

Das Skelett der Handwurzel und der Finger fehlt vollständig. Die Haut über den Gelenksenden des Vorderarmes ist schwach gerunzelt, jedoch nirgends adhärent oder narbig verändert.

Vom linken Vorderam ist nur ein $1 \frac{1}{2} \mathrm{~cm}$ langer Stummel vorhanden. Derselbe enthält als knöcherne Grundlage blos das Gelenksende der Ulna, während das Köpfehen des Radius nirgends zu spüren ist. Die Haut darüber zeigt eine ca. $1 / 2 \mathrm{~cm}$ lange, über dem Knochen versehiebliche Einziehung. Beugung und Streckung im linken Ellbogengelenk sind frei, die rohe Muskelkraft vorhanden. Auch links fehlen Handwurzel und Hand. Die Vorderarmknochen sind gerade soweit vorhanden, als die Ansätze der Oberarmmuseulatur reichen.

Figur 1.

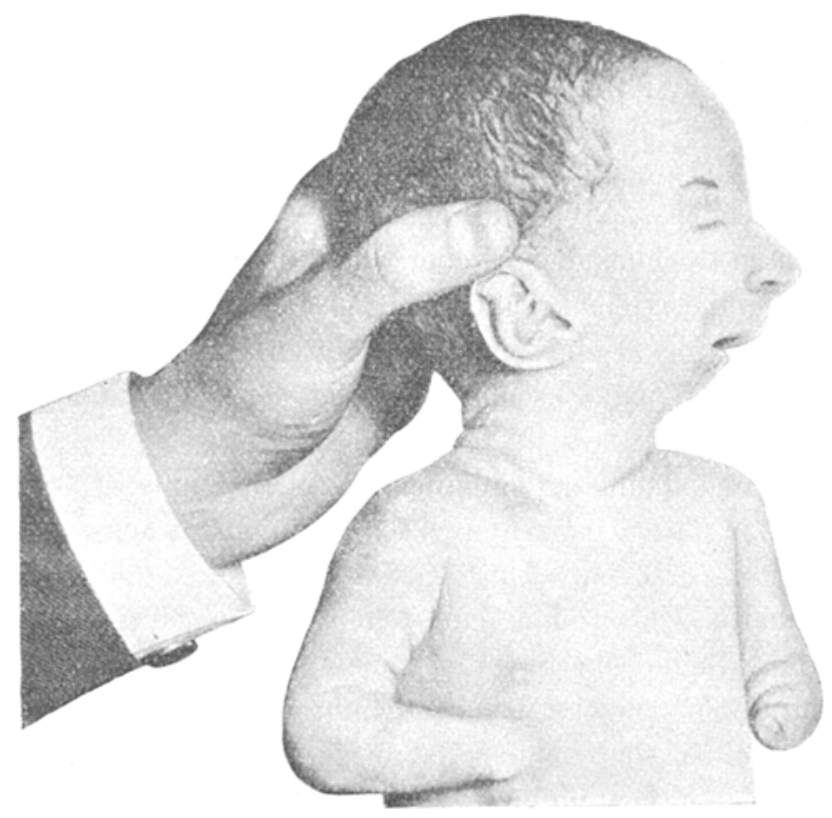

Röntgenbilder, welche vom Kopfe und den oberen Extremitäten durch Herm Dr. Holzknecht in liebenswürdiger Weise aufgenommen wurden, bestätigen die oben beschriebenen Verbältnisse.

Die Brust- und Bauchorgane zeigen keinerlei Abnormitäten: Das Herz ist normal gelagert, über den Ostien allenthalben reine 
Töne. Die Leber befindet sich an normaler Stelle. Die unteren Extremitäten sind gut und symmetrisch entwickelt. Die Haut der Analgegend war sagittal eingezogen, liess jedoch keine Oeffnung erkennen. Weder mit dem Katheter noch mit dünnen Sonden war eine Communication mit dem Darmrohr herzustellen. Das Kreuzbein endigt relativ hoch und breit. Der rechte Hode ist im Scrotum palpabel, der linke liegt im Leistenkanale.

Im Bereiche des centralen und peripheren Nervensystems fanden sich keinerlei erkennbare Störungen. Die bestehende Atresia ani wurde 2 Tage post partum durch Ineision und Herausnähen der Rectalschleimhaut an die äussere Bedeckung beseitigt, eine gleichzeitig bestehende Phimose durch Circumcision behoben.

Nachder das Kind keine Nahrung zu sich nehmen konnte, da die Saugbewegungen anfangs unmöglich schienen und auch die mittelst eines Löffels eingeflösste Milch beim rechten Mundwinkel wieder herausfloss, liess ich die Nahrung durch einen mit Glastrichter versehenen Nélatonkatheter. eingiessen, worauf dieselbe behalten wurde. Das Kind wurde nach 14 Tagen in häusliche Pflege entlassen und bei einer neuerlichen Vorstellung desselben nach weiteren zwei Wochen konnte ich dessen "relatives" Wohlbefinden constatiren. Fünf Wochen später jedoch brachte die Mutter das Kind in die Klinik, da dasselbe seit einigen Tagen alle Nahrung erbreche und an diarrhoeischen Stühlen leide. Das Kind war bei seiner Ankunft moribund und nach wenigen Stunden erfolgte der Exitus letalis. Dadurch war eine genaue Autopsie möglich gemacht worden, welche am 14. 6. ron Herrn Dr. Landsteiner ausgeführt wurde.

Der Befund ist folgender:

$50 \mathrm{~cm}$ lange, männliche Kindesleiche mit gut entwickeltem Panniculus. An der Haut keine Abnormitäten, ausser schuppenden Efflorescenzen der Kopf haut und Excoriationen in der Umgebung des Anus.

Schädelumfang $35 \mathrm{~cm}$. Am Gesicht zeigen sich die Lidspalten eng, die Bulbi etwas vorgedrängt. Die unteren Partien des Gesichtes hochgradig verbildet, die rechte Wangenhälfte abgeflacht. Die Wangengegend auf dieser Seite ganz ohne scharfe Grenze in die Submaxillargegend übergehend. Auch linkerseits die Submaxillargegend nach oben hin wenig scharf begrenzt. Die Mundöffnung zeigt fast eine runde Form; genauer ist sie derart beschaffen, dass die Unterlippe sehr kurz $(12 \mathrm{~cm})$ ist und dic Oberlippe auf ihr als Basis einen etwas spitzigen Bogen beschreibt, Der Helix rechterseits verbreitert.

Die oberen Extremitäten verbildet. Die rechte besitzt keine Hand und endet entsprechend dem unteren Ende des Vorderarmes stummel- 
förmig. Der linke Arm endigt gleichfalls stummelförmig im Bereiche des oberen Unterarmes.

Am Thorax und Abdomen keine wesentlichen Veränderungen.

Die Rückenhaut namentlich über dem Kreuzbeine, aber auch auf den oberen Antheilen des Rückens etwas behaart. Der Nabel bläschenförmig vorgewölbt. Am Orificium praeputii Narben.

Im Hodsnsacke beide Testikeln ${ }^{1}$ ), in der Ungebung der Anusoffnung Narbert.

An der Thymus keine auffallenden Abweichungen. Das Herz normal gelagert, äusserlich normal gebildet. Auch am eröffneten Herzen zeigen sich keine Bildungsanomalien.

Der Ductus Botalli zum grössten Theil verschlossen, von der Aorta aus auf eine Strecke von etwa $3 \mathrm{~mm}$ zugängig. In den hinteren Partien der rechten Lunge ist das Gewebe zum Theil luftleer, an einigen Stellen lässt sich eine trïbe, dickliche Flüssigkeit ausdrïcken. Andere Stellen zeigen eine feinköruige, leicht prominente Schnittfäche. Aehnliche Verhälnisse in der linken Lunge.

An der Lagerung der Eingeweide fällt keine wesentliche Veränderung auf. Die Leber von entsprechender Grösse, ziemlich blutreich, die Zeichnung wenig deutlich. Die Milz von normaler Grösse und Consistenz, Schnittfäche glatt, Follikel sichtbar.

Die Nebennieren zeigen keine Veränderungen. Das Nierenparenchym, namentlich in der Rinde erbleicht. Die Blasenschleimhant blass. Die Schleimhaut des Darmes im Ileum etwas aufgelockert, der Darm krümelige, gelbe Massen enthaltend. Der Schleimhaut haftet etwas Schleim an. Das Colon stark erweitert, breiige, gelbe Inbaltmassen enthaltend. Schleimhaut blass und glatt.

Die genauere Präparirung hatte nun folgendes Ergebniss: Vom Unterkiefer zeigt sich nach dem Abpräpariren der Haut die linke Seite erhalten, von der rechten Seite ist, von der Mittellinie an gerechnet, nur ein $12 \mathrm{~mm}$ langes Stück, welches nach rechts zu mit stumpfer Spitze endet, vorhanden. Dareh die Muskeln hindurch lässt sich in der Gegend des Kiefergelenkes rechterseits ein bohnengrosses Knochenstückchen tasten.

Die Zunge ist dünn, sie ist auf der linken Seite wohlgeformt, auf der rechten Seite scheint sie abgeflacht, hat keine freie Unterfläche und keinen freien Rand; es geht vieJmehr die Schleimhaut vom Zungenrücken, nur von einer seichten, dem Zungenrande entsprechenden Furche durchzogen, in die Mucosa der Wange über. Die Muskeln und die übrigen Gebilde, einschliesslich der Submaxillardrüse der linken Seite zeigen keine auffallenden Veränderungen. Auf der rechten Seite ist eine Submaxillardriise makroskopisch nicht nachweisbar.

1) Offenbar war der linke T'estikel erst während des extrauterinen Lebens vollständig descendirt. 
Die Muskeln zeigen mannigfache Verbildungen. Vom Jochbogen entspringen Masseterfasern, in geringerem Ausmaasse angelegt als links, die zum Theil beim Abpräpariren der Haut durchtrennt werden, zum Theil sich einem voluminösen Muskelbauche anschliessen, welcher eine zum Zungenbeine hinziehende Fortsetzung des M. temporalis darstellt. Dieser Muskelbauch lässt zwei runde Bündel erkennen, die sich aber nicht sehr deutlich isoliren lassen. Der M. biventer ist in seinem hinteren Antheile ebenso wie der M. stylo-hyoideus völlig ausgebildet; nach vorn zu geht der vordere Bauch an seinen normalen Ansatzpunkt, lässt sich aber nicht von den Fasern des darunter liegenden, in der Nachbarschaft der Mittellinie auch rechterseits nachweisbaren, vom Zungenbein gegen den Kiefer aufsteigenden M. mylo-byoidens trennen.

Bei der Präparation von hinten her zeigt sich ein aus der Fossa pterygoidea entspringendes Bündel von Muskelfasern, von dem ein Theil nach abwärts fibrös gegen das Ligamentum stylohyoideum, ein anderer Theil gegen das erwàhnte Knochenstückchen am Kiefergelenke hinzieht. Diese dem M. pterygoideus entsprechenden Bündel sind im Vergleiche mit den M. pterygoid. der normalen Seite sehr schwach ausgebildet.

Es zeigte sich demnach, dass zwar alle Muskeln der oberflächlichen und tiefen Schichten vorhanden waren, in Folge der fehlenden Insertionspunkte am Unterkiefer jedoch theils ganz von der Norm abweichende Verlaufsrichtungen nehmen mussten, theils eine Inactivitätsatrophie aufwiesen, die an manchen Muskelgruppen so hochgradig war, dass man ihr Entstehen jedenfalls in das Stadium der intrauterinen Entwickelung zurückverlegen muss. Auffallend ist das vollständige Fehlen einer Submaxillardrüse rechterseits, da auch die histologisehe Untersuchung einzelner Gewebsstücke, die als ein atrophischer Rest einer solchen hätten angesehen werden können, ein negatives Resultat ergab.

Bei der anatomischen Präparation der Muskeln der oberen Extremitäten zeigte sich beiderseits die Sehultergürtelmuskulatur völlig normal entwickelt. Die Muskulatur des rechten Oberarms bot gleichfalls normale Verhältnisse. Am rechten Vorderarm erwies sich der M. flexor sublimis insotern von der Norm abweichend, als er, nur eine kurze Strecke weit fleischig entwickelt, sodann in eine dünne Aponeurose überging, welche den Nervus medianus überlagerte.

Auf der linken Seite fand sich für die Streckmuskeln des 
Vorderarmes ein Caput commune ausgebildet, welches in der Haut endete, bevor es sich in einzelne Muskelgruppen aufgelöst hatte.

Von den Knochen war zwar der Diaphysenansatz der Ulna yorhanden, das Gelenkende des Radius fehlte jedoch völlig, welcher Befund mit den früher aufgenommenen Röntgenbildern sich deckte.

Die histologische Verarbcitung des Centralnervensystems, welehe Herr Dr. Zappert übernahm und deren Ergebnisse er demnächst gesondert besprechen wird, ergab den interessanten Befund einer ausgebreiteten Hydromyelie, welche das ganze Cervicalmark einnahm und zwischen 2. und 5. Cervicalnerven die grösste Ausdehnung erreichte, so dass bier das Lumen des Centralkanales ein Drittel der Oberfäche des Querschnittes ausmachte. Der Centralkanal selbst hat eine rhomboide Gestalt mit einem rach hinten ausladenden zapfenförmigen Vorsprung, welcher bei Missbildungen typisch vorkommen soll.

Figur 2.

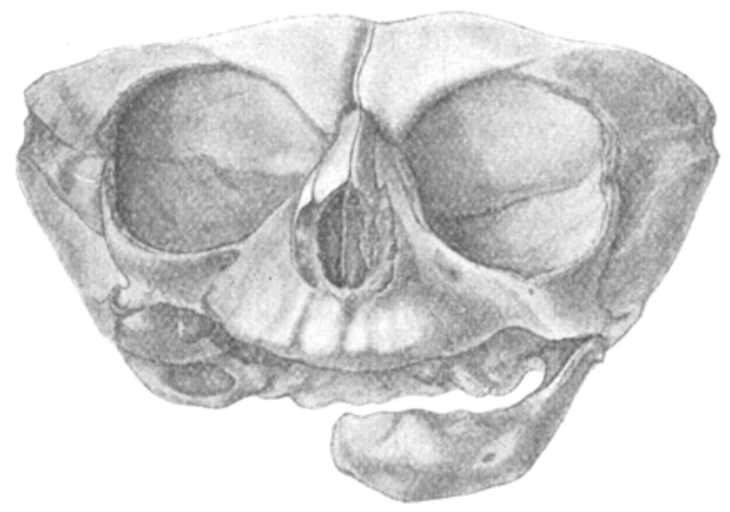

Nach unten gegen das Dorsalmark zu wird die Lrweiterung des Centralkanales allmälig geringer, um im Iendenmarke einem normalen Lumen Platz zu machen. In den Strängen des Rückenmarkes sowohl als auch in der Anlage der Ganglienzellen waren keine Abweichungen vom Tormalen za erkennen, so dass sich auf Grund der Befunde im Rückenmarke kein directer Zusammenhang mit den Defecten an den oberen Extremitäten, noch weniger mit den Verbildungen im Bereiche des Gesiehts aussprechen lässt. - Schliesshich wurde auch der Schädel skelettirt und der Betund in vorstehender Abbildung (Fig. 2) festgehalten. Man sieht deut- 
lich die Verbildung der rechtsseitigen Orbita, welche sich als eine Verkürzung des sagittalen Durchmessers documentirt; ferner die stumpfe Endigung der linken Unterkieferhälfte nur wenig rechts von der Medianlinie und das Vorhandensein eines ungefähr Jinsengrossen Knochenstückes in der nur durch eine Abflachung des Knochens gekennzeichneten Gelenkgrabe rechterseits, welcher wohl als primäre Anlage des Unterkiefer-Gelenkkopfes aufzufassen ist.

Ein frontaler Durchschnitt durch den vorderen Antheil des Unterkieferknochens zeigte, dass die Zahnsäckchen zweier Schneideund des Eckzahns angelegt sind, so dass von der rechten Seite keinerlei Zahnanlage vorhanden ist.

Wenn wir nun die verschiedenen Hemmungsbildungen bei diesem Individuum betrachten, so scheint eine Sichtung derselben nothwendig in solche, welche untereinander in einem gewissen $\mathrm{Zu}-$ sammenhang stehen und solche, welche unabhängig von einander entstanden gedacht werden können. Zu letzteren gehören neben der Hydromyelie des Rückenmarks die rudimentäre Entwickelung der oberen Extremitäten, welche als Perobrachius zu bezeichnen wäre, und die Atresia ani. Die beiden letaten Anomalien sind relativ nicht zu selten vorkommende Befunde und auch in der Literatur vielfach beschrieben.

Erhöhtes Interesse beanspruchen jedoch die Entwickelungsstörungen im Bereiche des Gesichtes, unter denen ein Zusammenhang zu bestehen scheint, so dass wir die knöchernen und muskulären Verbildungen als secundär abhängig von dem Mangel der rechten Unterkieferhälfte auffassen können und auch der scheinbar vollständige Defect der rechten Unterkiefer-Speicheldrüse erklärt erscheint.

Eine Durchsicht der diesbezüglichen Literatur ergab, dass Fälle, bei denen sich angeborenerweise entweder ein vollständiger Yangel beider Unterkieferhälften oder eine abnorme Kleinheit des Unterkieferknochens fand, in geringer Zahl bekannt sind.

So beschreibt G. Brann ${ }^{1}$ ) ein neugeborenes Kind, bei dem kein Bestandtheil des Unterkiefers vorhanden war. Gleichzeitig fand sich Synotie, also Verwachsung beider Ohren in der Mittellinie. Er bezeichnet den Fall als Agnathus. Arnold²) beschreibt

1) Ein Fall von Agnathus. Zeitschrift der k. k. Gesellschaft der Aerzte. XI. Wien. 1855.

2) Beschreibung einer Missbildung mit Agnathie and Hydropsie der gemeinsamen Schland-Trommelhöhle. Virchow's Archiv. Bd. 38. 
eine Missbildung mit Agnathie und giobt an, dass Unterkiefer und Zungenbein vollständig fehlten. Einen ähnlichen Fall erwähnt Panl'1).

Fine Zusammenstellung von 14 Fällen angeborener Kleinheit und Asymmetrie des Unterkiefers giebt Winckel ${ }^{2}$ ). Langenbeck ${ }^{3}$ ) beschreibt bei einem 17 jährigen Mann eine angeborene Kleinheit des Unterkiefers und erwähnt, dass diese Bildungsanomalie bei Thieren, namentlich beim Lamm relativ häufiger vorkomme. Förster ${ }^{4}$ ) bespricht einen ausgetragenen Fötus, bei dem der Unterkiefer, abnorm klein, hinter dem Oberkiefer so zurücksteht, dass der vordere Stand seines Alveolarfortsatzes an den hinteren Rand desselben Fortsatzes des Oberkiefers stösst. Auch A. Marc ${ }^{5}$ ) erwähnt eine siebenmonatliche Frucht, welche nach 3 Tagen gestorben war, bei welcher der Unterkiefer entwickelt, aber in allen Dimensionen in der Entwickelung zurückgeblieben war. Ferner stellte $W_{0} f_{f}{ }^{b}$ ) in der Berliner medicinischen Gesellschaft 2 Fälle von angeborener Mikrognathie vor und Koblank ${ }^{7}$ ) einen gleichen Fall, bei dem gleichzeitig Perobrachius bestand. Endlich giebt Loren $z^{8}$ ) aus der Klinik Albert eine Zusammenstellung über einige Fälle von Mikrognathie, unter denen er zwei als angeboren bezeichnet.

Von Fällen jedoch, welche mit dem unserigen nur eine gewisse Aehnlichkeit zeigen, konnte ich nur zwei finden und zwar einen Fall von Virchow ${ }^{9}$ ) aus dem Jahre 1864, in welchem der

1) Bullet. de la Societé de Gand. 1857. Fevrier. Citirt nach Winckel.

2) Aetiologische Untersuchungen über einige sehr seltene fötale Miss* bildungen. Münchener medicinische Wochenschrift. 1896. No, 18.

3) Angeborene Kleinheit des Unterkiefers. Archiv für klin. Chirurgie. I.

4) Mittheilungen aus der pathologisch-anatomischen Anstalt zu Würzburg. Würzburger medicinische Zeitschrift. 1862.

5) Ein seltener Fall von Mikrognathie mit Mundbodenspalte. Arbeiten aus dem Gebiete der patholog. Anatomie und Bakteriologie. Tübingen. 1891 bis 1892 .

6) Berliner medicinische Gesellschaft. Sitzung vom 3. 3. 1897.

7) Geburtshülflich-gynäkologische Gesellschaft zu Berlin. Sitzung vom 25. 10. 1895 .

8) Kasuistische Beiträge zur Kenntniss der Mikrognathie. Deutsche Zeitschrift für Chirurgie,

9) Ueber Missbidungen am Ohre und im Bereiche des 1. Kiemenhogens. Virchow's Archiv. 1864. 
rechte Unterkiefer in der Gegend der Backenzähne ganz plötzlich mit einem dicken, abgerundeten Rande aufhörte, sodass also Processus coronoides und condyloideus fehlten; an das Knochenende schloss sich ein derbes Ligament, welches sich in der Gegend des Tuberculum articulare an das Scbläfenbein inserirte. Ein 2. Fall

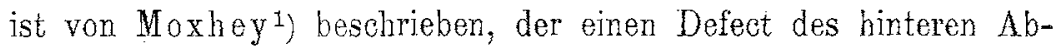
schnittes der rechten Unterkieferhälfte vom 1. Backenzahne ab findet. Der Kiefertheil war auch hier durch einen ziemlich festen Gewebsstrang ersetzt. Ein Defect jedoch, welcher sowohl beide Fortsätze als auch den aufsteigenden Ast und das Corpus mandibulae in sich begreift, ist meines Wissens bis jetzt noch nicht bekannt geworden und so wäre diese Hemmungsbildung als Hemignathie zu bezeichnen.

Wenn wir nun nach der Ursache dieser Entwickelungsstörung fragen, so kommen hierbei zwei Möglichkeiten der Erklärung in Betracht: Entweder handelt es sich um einen primären Defect in der ersten Anlage des Kopfskelettes oder es sind während der intrauterinen Entwickelung des Fötus mechanische Momente in Action getreten, welche die normale Ausbildurg der Theile verhindert baben. Als solche secundäre Störungen kämen in Betracht eine auffallend geringe Fruchtwassermenge, ein frühzeitiges Verwachsen der Amnionkopfkappe oder Umschnürungen einzelner Theile durch sogenannte amniotische oder Simonart'sche Bänder.

Letztere Erklärung hat auf den ersten Blick viel mehr Wabrscheinlichkeit für sich und auch die meisten Autoren neigen sich dieser Ansicht zu; so namentlich Winckel, welcher seine Fälle als "fötale Compressionsatrophie" bezeichnet. Als Beweis für die Richtigkeit dieser Auffassung führt er an, dass die Knochen und Weichtheile doch nachzuweisen sein müssten, was ihm auch in sieben von 12 Fällen gelang. Ferner spricht er tiefe Furchen oder sogar geradezu Einschnürungen mit Faltenbildung nach dem hinteren Ende des Oberkiefers hin, die sich in einigen seiner Fälle fanden, als Beweis an, dass ron aussen einwirkende bandartige Einschnürungen die Ursache der Veränderung sind.

Virchow spricht nur von irritativen Störungen im Bereiche des 1. Kiemenbogens. Hingegen findet $\mathrm{Braun}$ die muthmaassliche Ursache nur in der Entwickelungsweise des Unterkiefers und glaubt,

1) Citirt nach Trendelenburg, Deutsche Chirurgie. Lieferung 33. 1. 
dass "keine zur Bildung des Unterkiefers nothwendige Blastemablagerung stattgefunden hat und demnach auch die Entwickelung des Unterkiefers unterblieben ist." Auch Marchand1, erwähnt, dass völlige Defecte einzelner Theile des Skelettes vorkommen, ohne dass sich eine andere Ursache dafür auffinden lässt, als ein "Mangel der Anlage".

Was nun unseren Fall anlangt, so spricht die Entwickelungsstörung an den Extremitäten und namentlich die Asymmetrie derselben allerdings für secundär einwirkende. Ursachen, also vielleicht amniotische Umschnürungen. Nicht angängig ist es jedoch die Befunde im Rückenmarke, die Atresia ani, die Combination mit den Extremitätenverkrümmungen, kurz die Multiplicität der Hemmungsbildungen auf dieselbe Weise erklären zu wollen. Ferner spricht anch das einseitige Auftreten des Defectes am Unterkiefer gegen die Annahme amniotischer Umschnürungen. Auch fehlte jede Andeutung einer Einschnürung in der unteren Gesichtsbälfte, es fanden sich keine Bindegewebsstränge an Stelle des fehlenden Unterkiefers, nirgends Furchen, Einziehungen oder Narben in der Haut. Die Placenta und Eihäute zeigten vollständig normale Verhältnisse, nirgends waren strangartige Verdicknngen vorhanden, welche man als Reste amniotischer Verwachsungen hätte auffassen können. Schliesslich war auch die Menge des Fruchtwassers bei der Geburt eher vermehrt als vermindert gewesen. Dass sich am skelettirten Schädel thatsächlich die Anlage eines Gelenkköplchens vorfand, spricht nicht für eine secundäre Compressionsatrophie im Sinne Winckels, da sich der Unterkiefer aus vielen Knochenkernen entwickelt, die auch unabhänging von einander zur Ausbildung kommen könnten.

Wenn man nun bedenkt, dass die Entwickelungsstörung in ein sehr frühzeitiges Stadium des embryonalen Lebens gefallen sein muss, da sich aus dem Unterkieferfortsatze des ersten Kiemenbogens schon in der neunten Woche der Unterkiefer bildet, so hat die Annahme wohl mehr Wahrscheinlichkeit für sich, dass es sich in diesem Falle um einen primären Defect in der Anlage, um einen Mangel an Bildungsmaterial gehandelt haben dürfte, welehe zu dem eigenthïmlichen Abweichen von der Norm geführt haben.

1) Missbildungen. Eulenburg's Encyklopädie. 
Eine gewisse Bedeutung gewimnt unser Fall auch dadureh, dass das Individuum lebend und reif zur Welt kam, dass es somit klinisch beobachtet werden konnte, während bisher alle ähnlichen Fälle entweder todtgeboren wurden oder unmittelbar post partum starben. Schliesslich ist durch diesen Fall auch gleichsam ein Uebergangsstadium zwischen jenen Bildern fixirt, welche bisher als Agnathie und Mikrognathie beschrieben worden sind.

Man wird also auch in Zukunft nicht alle Fälle von angeborener Missbildung auf secundär einwirkende Ursachen beziehen können und bei einzelnen als Erklärung auf Defecte in der primären Keimanlage zurückgreifen müssen. 